\title{
Reidemeister orbit sets
}

\author{
by \\ Boju Jiang (Beijing), Seoung Ho Lee (Daejeon) \\ and Moo Ha Woo (Seoul)
}

\begin{abstract}
The Reidemeister orbit set plays a crucial role in the Nielsen type theory of periodic orbits, much as the Reidemeister set does in Nielsen fixed point theory. Extending Ferrario's work on Reidemeister sets, we obtain algebraic results such as addition formulae for Reidemeister orbit sets. Similar formulae for Nielsen type essential orbit numbers are also proved for fibre preserving maps.
\end{abstract}

0. Introduction. Nielsen fixed point theory has been extended to a Nielsen type theory of periodic orbits [6, Section III.3]. In fixed point theory, the computation of the Nielsen number often relies on the knowledge of the Reidemeister set, that is, the set of Reidemeister conjugacy classes in the fundamental group. Ferrario [2] made an algebraic study of the Reidemeister set in relation to an invariant normal subgroup. He obtained addition formulae for Reidemeister numbers, and applied them to the Nielsen number of fibre preserving maps. Our aim in this paper is similar, but in the more complicated setting of periodic orbits: to study the Reidemeister orbit set of a group endomorphism in relation to an invariant normal subgroup, to obtain addition formulae for Reidemeister orbit numbers, and as application, to find addition formulae for Nielsen type essential orbit numbers of fibre preserving maps.

Given a group endomorphism $f: G \rightarrow G$, the Reidemeister set of $f$, denoted by $\mathcal{R}(f)$, is the set of orbits of the left action of $G$ on $G$ via $\gamma \stackrel{g}{\mapsto}$

2000 Mathematics Subject Classification: Primary 55M20.

Key words and phrases: Reidemeister sets, Reidemeister orbit sets, Nielsen type essential $n$-orbit numbers, essential $n$-orbit representatives.

This work was started during the visit of the first author to Korea University, Seoul, in Spring 2003, under the Brain Pool Program of KOFST. He has been partially supported by a MOSTC grant.

The third author is supported by a Korean Research Foundation program of the year 2002 . 
$g \gamma f\left(g^{-1}\right)$, and its cardinality is the Reidemeister number $R(f)$ of $f$. When $f$ is the homomorphism induced by a map $X \rightarrow X$ on the fundamental group $\pi_{1}(X), R(f)$ is an upper bound for the Nielsen number $N(f) ; N(f)$ is usually the minimal number of fixed points in the homotopy class of the map. For many spaces, such as nil and solvmanifolds, the computation of the Nielsen number reduces to that of $R(f)$ (see [3]).

For a given integer $n>0, f$ acts on the Reidemeister set $\mathcal{R}\left(f^{n}\right)$ of the $n$th iterate $f^{n}$. An orbit of this action is called a Reidemeister orbit, the set of all such orbits is the Reidemeister orbit set $\mathcal{R O}^{(n)}(f)$. When $f$ is the homomorphism induced by a map $X \rightarrow X$ on $\pi_{1}(X)$, its cardinality $\sharp \mathcal{R O} \mathcal{O}^{(n)}(f)$ is an upper bound for the number of essential $n$-orbits, the latter being a lower bound for the number of $n$-orbits in the homotopy class.

Let $f: G \rightarrow G$ be a group endomorphism. If $H \subset G$ is an $f$-invariant normal subgroup and $\bar{G}=G / H$, then the short exact sequence $1 \rightarrow H \stackrel{i}{\rightarrow}$ $G \rightarrow \bar{G} \rightarrow 1$ induces an exact sequence (in the category of pointed sets)

$$
\mathcal{R} \mathcal{O}^{(n)}\left(f_{H}\right) \stackrel{i_{*}}{\rightarrow} \mathcal{R} \mathcal{O}^{(n)}(f) \rightarrow \mathcal{R} \mathcal{O}^{(n)}(\bar{f}) \rightarrow 1
$$

of Reidemeister orbit sets. We shall find conditions for the function $i_{*}$ to be injective. Under certain conditions, we will prove an addition formula of the form

$$
\sharp \mathcal{R} \mathcal{O}^{(n)}(f)=\sum_{j \in \mathcal{R} \mathcal{O}^{(n)}(\bar{f})} \sharp \mathcal{R} \mathcal{O}^{\left(m_{j}\right)}\left(\theta_{j}^{\prime}\right),
$$

where $m_{j}=n / \ell_{j}, \ell_{j}$ being the length of the orbit $j$, and $\theta_{j}^{\prime}: H \rightarrow H$ is a twisted version of $f_{H}^{\ell_{j}}$.

Turning to the topological context, we consider a fibre preserving map $f: E \rightarrow E$ of a Hurewicz fibration $p: E \rightarrow B$ of compact ANR's. It induces a map $\bar{f}: B \rightarrow B$. Let $K$ be the kernel of the homomorphism $j_{*}$ : $\pi_{1}\left(F_{b}\right) \rightarrow \pi_{1}(E)$ induced by the inclusion of the fibre. Denote by $E O^{(n)}(f)$ the number of essential $n$-orbit classes of $f$, and by $E O_{K}^{(m)}$ the number of mod $K$ essential orbit classes on a fibre. Under suitable conditions, we have an addition formula of the form

$$
E O^{(n)}(f)=\sum_{b \in \xi} E O_{K}^{(m)}\left(h_{b}\right),
$$

where the summation runs over a set $\xi$ of essential $n$-orbit representatives for $\bar{f}, \ell$ is the length of the essential $\bar{f}$-orbit class containing $b, m=n / \ell$, and $h_{b}: F_{b} \rightarrow F_{b}$ is a variant of $f^{\ell} \mid F_{b}$.

The paper consists of two sections. In the first section we show our results on Reidemeister orbit sets using the method of Ferrario's algebraic results in [2] on the Reidemeister sets. In the second section we apply them to fibre preserving maps. 
For the basics of Nielsen fixed point theory, the reader is referred to [1] and $[6]$.

1. The Reidemeister orbit set. Let $f: G \rightarrow G$ be a group endomorphism. The Reidemeister set of $f$, denoted by $\mathcal{R}(f)$, is the set of equivalence classes for the following Reidemeister equivalence relation in $G: \gamma, \gamma^{\prime} \in G$ are equivalent if and only if $\gamma^{\prime}=g \gamma f\left(g^{-1}\right)$ for some $g \in G$. The Reidemeister class of $\gamma \in G$ will be written $[\gamma]_{f}$.

If $H \subset G$ is an $f$-invariant normal subgroup, then the short exact sequence

$$
1 \rightarrow H \stackrel{i}{\rightarrow} G \stackrel{q}{\rightarrow} \bar{G} \rightarrow 1,
$$

where $\bar{G}=G / H$, and $i: H \rightarrow G$ and $q: G \rightarrow \bar{G}$ are the inclusion and quotient homomorphisms, induces an exact sequence (in the category of pointed sets)

$$
\left(\mathcal{R}\left(f_{H}\right),[1]_{f_{H}}\right) \stackrel{i_{*}}{\rightarrow}\left(\mathcal{R}(f),[1]_{f}\right) \stackrel{q_{*}}{\rightarrow}\left(\mathcal{R}(\bar{f}),[1]_{\bar{f}}\right) \rightarrow 1
$$

of Reidemeister sets, where $\mathcal{R}\left(f_{H}\right)$ is the Reidemeister set of the restriction map $f_{H}: H \rightarrow H$, and $\mathcal{R}(\bar{f})$ is the Reidemeister set of the induced map $\bar{f}: \bar{G} \rightarrow \bar{G}$.

The function $i_{*}$ is not injective in general. In Davide Ferrario's paper [2], an $f$-invariant normal subgroup $T_{f}(K)$ in $H$ is identified so that under certain conditions, the image $i_{*} \mathcal{R}\left(f_{H}\right)$ is in one-to-one correspondence with the Reidemeister set $\mathcal{R}\left(\widehat{f}_{H}\right)$ of the induced map $\widehat{f}_{H}: \bar{H} \rightarrow \bar{H}$, where $\bar{H}=$ $H / T_{f}(K)$.

Definition 1.1. Let $n>0$ be a given integer. Then $f$ acts on the Reidemeister set $\mathcal{R}\left(f^{n}\right)$ by $[\gamma]_{f^{n}} \stackrel{f}{\mapsto}[f(\gamma)]_{f^{n}}$. The $f$-orbit of a Reidemeister class $[\gamma]_{f^{n}}$ will be called a Reidemeister $n$-orbit, denoted by $[\gamma]_{f}^{(n)}$. The set of all such Reidemeister $f$-orbits will be called the Reidemeister $n$-orbit set of $f$, denoted by $\mathcal{R O}^{(n)}(f)$.

The length of the orbit $[\gamma]_{f}^{(n)}$ is the smallest integer $\ell>0$ such that $[\gamma]_{f^{n}}=\left[f^{\ell}(\gamma)\right]_{f^{n}}$. Clearly $\ell$ divides $n$.

REMARK 1.2. $\mathcal{R O}^{(n)}(f)$ is the set of equivalence classes in $G$ of the following equivalence relation: $\gamma, \gamma^{\prime} \in G$ are equivalent if and only if

$$
\gamma^{\prime}=g f^{i}(\gamma) f^{n}\left(g^{-1}\right) \quad \text { for some } i \geq 0 \text { and } g \in G .
$$

For any integer $n>0$, there is an exact sequence (in the category of pointed sets)

$$
\left(\mathcal{R O}^{(n)}\left(f_{H}\right),[1]_{f_{H}}^{(n)}\right) \stackrel{i_{*}}{\rightarrow}\left(\mathcal{R} \mathcal{O}^{(n)}(f),[1]_{f}^{(n)}\right) \stackrel{q_{*}}{\rightarrow}\left(\mathcal{R O}^{(n)}(\bar{f}),[1]_{\bar{f}}^{(n)}\right) \rightarrow 1
$$


of Reidemeister orbit sets, where $\mathcal{R} \mathcal{O}^{(n)}\left(f_{H}\right)$ is the Reidemeister orbit set of the restriction map $f_{H}: H \rightarrow H$, and $\mathcal{R O}^{(n)}(\bar{f})$ is the Reidemeister orbit set of the induced map $\bar{f}: \bar{G} \rightarrow \bar{G}$. For any $\gamma \in G$ we shall always write $\bar{\gamma}:=q(\gamma)$, so that $q_{*}\left([\gamma]_{f^{n}}\right)=[\bar{\gamma}]_{\bar{f}^{n}}$ and $q_{*}\left([\gamma]_{f}^{(n)}\right)=[\bar{\gamma}]_{\bar{f}}^{(n)}$.

We now consider the algebraic results in [2], applied to the endomorphism $f^{n}: G \rightarrow G$. Since the endomorphisms $f_{H}, \bar{f}$ etc. are all induced by $f$, the maps established between their Reidemeister sets $\mathcal{R}\left(f^{n}\right)$ etc. are equivariant under the $f$-action, so they induce maps between the Reidemeister orbit sets $\mathcal{R O}^{(n)}(f)$ etc.

Definition 1.3. Suppose $K \subset G$ is an $f$-invariant subgroup of $G$ and

$$
K H=q^{-1} \operatorname{Fix}\left(\bar{f}^{n}\right) .
$$

Such $K$ exist, for example the subgroup $q^{-1} \operatorname{Fix}\left(\bar{f}^{n}\right)$ itself. Let $[K, H]$ denote the subgroup of $G$ generated by all $k h k^{-1} h^{-1}$ such that $k \in K$ and $h \in H$. Let $K^{G}$ denote the smallest normal subgroup of $G$ containing $K$. Define

$$
O_{f^{n}} K:=\left\{k f^{n}\left(k^{-1}\right) \mid k \in K\right\} .
$$

Let

$$
T_{f^{n}}(K):=\left[K^{G}, H\right] \cup O_{f^{n}} K
$$

be the smallest subgroup of $G$ containing $\left[K^{G}, H\right]$ and $O_{f^{n}} K$.

Ferrario's proofs ([2, pp. 5-7] with $f$ replaced, whenever it occurs, by $f^{n}$ ) establish the following results 1.4, 1.5 and 1.6.

Proposition 1.4. The subgroup $T_{f^{n}}(K)$ is normal in $H$ and $f$-invariant, and it equals $\left\{\alpha k f^{n}\left(k^{-1}\right) \mid \alpha \in\left[K^{G}, H\right], k \in K\right\}$.

Lemma 1.5. For any $f$-invariant subgroup $K$ of $G$ such that

$$
K H=q^{-1} \operatorname{Fix}\left(\bar{f}^{n}\right),
$$

there exists a surjection

$$
A: q_{*}^{-1}\left([1]_{\bar{f}}^{(n)}\right)=i_{*} \mathcal{R} \mathcal{O}^{(n)}\left(f_{H}\right) \rightarrow \mathcal{R} \mathcal{O}^{(n)}\left(f_{H}^{\prime}: H / T_{f^{n}}(K) \rightarrow H / T_{f^{n}}(K)\right)
$$

defined by $A\left([h]_{f}^{(n)}\right):=[p(h)]_{f_{H}^{\prime}}^{(n)}$ for all $h \in H$, where $p$ is the projection $p: H \rightarrow H / T_{f^{n}}(K)$, and $f_{H}^{\prime}$ is induced by $f_{H}$. Moreover, $A$ is injective whenever $\mathcal{R O}^{(n)}(f)=\mathcal{R} \mathcal{O}^{(n)}\left(\widehat{f}: G /\left[K^{G}, H\right] \rightarrow G /\left[K^{G}, H\right]\right)$.

Corollary 1.6. If $\operatorname{Fix}\left(\bar{f}^{n}\right)=\{1\}$, then

$$
i_{*}: \mathcal{R O}^{(n)}\left(f_{H}\right) \rightarrow q_{*}^{-1}\left([1]_{\bar{f}}^{(n)}\right) \subset \mathcal{R} \mathcal{O}^{(n)}(f)
$$

is a bijection. 
How do we deal with $q_{*}^{-1}\left([\bar{\alpha}]_{\bar{f}}^{(n)}\right) \subset \mathcal{R} \mathcal{O}^{(n)}(f)$ for an arbitrary $\alpha \in G ?$

LEMmA 1.7. Let $n>0$ and $\alpha \in G$. Suppose the orbit $[\bar{\alpha}]_{\bar{f}}^{(n)} \in \mathcal{R O}^{(n)}(\bar{f})$ has length $\ell$, and let $m:=n / \ell$. We have a commutative diagram of exact sequences in the category of pointed sets:

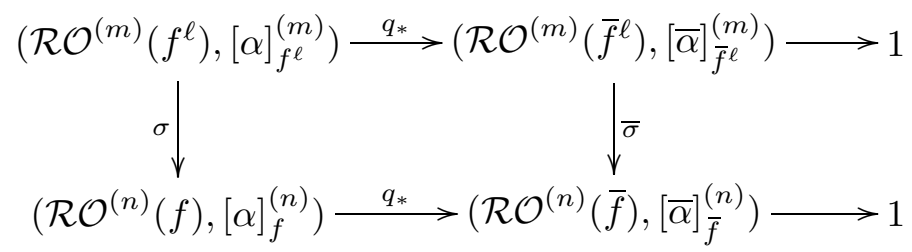

where the vertical maps $\sigma$ and $\bar{\sigma}$ are induced by inclusions, and they are surjective. Furthermore, $\sigma$ restricts to a bijection

$$
\sigma: q_{*}^{-1}\left([\bar{\alpha}]_{\bar{f} \ell}^{(m)}\right) \rightarrow q_{*}^{-1}\left([\bar{\alpha}]_{\bar{f}}^{(n)}\right) .
$$

Proof. First we will show that $\sigma\left(q_{*}^{-1}\left([\bar{\alpha}]_{\bar{f}^{\ell}}^{(m)}\right)\right) \subset q_{*}^{-1}\left([\bar{\alpha}]_{\bar{f}}^{(n)}\right)$. If $[\beta]_{f^{\ell}}^{(m)} \in$ $q_{*}^{-1}\left([\bar{\alpha}]_{\bar{f} \ell}^{(m)}\right)$, then $[\bar{\beta}]_{\bar{f} \ell}^{(m)}=[\bar{\alpha}]_{\bar{f} \ell}^{(m)}$, and hence

$$
\bar{\beta}=\bar{\gamma}\left(\bar{f}^{\ell}\right)^{i}(\bar{\alpha})\left(\bar{f}^{\ell}\right)^{m}\left(\bar{\gamma}^{-1}\right)=\bar{\gamma} \bar{f}^{\ell i}(\bar{\alpha}) \bar{f}^{n}\left(\bar{\gamma}^{-1}\right)
$$

for some $i \geq 0$ and $\bar{\gamma} \in \bar{G}$. Therefore $q_{*} \circ \sigma\left([\beta]_{f^{\ell}}^{(m)}\right)=[\bar{\alpha}]_{\bar{f}}^{(n)}$.

Suppose $[\beta]_{f}^{(n)} \in q_{*}^{-1}\left([\bar{\alpha}]_{\bar{f}}^{(n)}\right)$. Then $\bar{\alpha}=\bar{\eta} \bar{f}^{i}(\bar{\beta}) \bar{f}^{n}\left(\bar{\eta}^{-1}\right)$ for some $i \geq 0$ and $\bar{\eta} \in \bar{G}$. There exists $\left[f^{i}(\beta)\right]_{f^{\ell}}^{(m)} \in \mathcal{R} \mathcal{O}^{(m)}\left(f^{\ell}\right)$ such that

$$
\left[\bar{f}^{i}(\bar{\beta})\right]_{\bar{f}^{\ell}}^{(m)}=[\bar{\alpha}]_{\bar{f}^{\ell}}^{(m)} \text { and } \quad \sigma\left(\left[f^{i}(\beta)\right]_{f^{\ell}}^{(m)}\right)=[\beta]_{f}^{(n)} .
$$

Thus the restriction map $\sigma$ is surjective.

Clearly

$$
q_{*}^{-1}\left([\bar{\alpha}]_{\bar{f}^{\ell}}^{(m)}\right)=\bigcup_{[\beta]_{f}^{(n)} \in q_{*}^{-1}\left([\bar{\alpha}]_{\bar{f}}^{(n)}\right)}\left(\sigma^{-1}\left([\beta]_{f}^{(n)}\right) \cap q_{*}^{-1}\left([\bar{\alpha}]_{\bar{f}^{\ell}}^{(m)}\right)\right),
$$

and

$$
\sigma^{-1}\left([\beta]_{f}^{(n)}\right)=\left\{[\beta]_{f^{\ell}}^{(m)},[f(\beta)]_{f^{\ell}}^{(m)},\left[f^{2}(\beta)\right]_{f^{\ell}}^{(m)}, \ldots,\left[f^{\ell-1}(\beta)\right]_{f^{\ell}}^{(m)}\right\}
$$

By definition of the length $\ell$ of the orbit $[\bar{\alpha}]_{\bar{f}}^{(n)}$, each orbit $\left[\bar{f}^{i}(\bar{\alpha})\right]_{\bar{f}^{\ell} \ell}^{(m)}$ consists of a single Reidemeister class $\left[\bar{f}^{i}(\bar{\alpha})\right]_{\bar{f}^{n}}$, and the orbits $\left\{\left[f^{i}(\beta)\right]_{f^{\ell}}^{(m)} \mid\right.$ $0 \leq i<\ell\}$ are different from each other. If some element $\left[f^{j}(\beta)\right]_{f^{\ell}}^{(m)}$ of $\sigma^{-1}\left([\beta]_{f}^{(n)}\right), 0 \leq j<\ell$, is in $q_{*}^{-1}\left([\bar{\alpha}]_{\bar{f}^{\ell}}^{(m)}\right)$, we have 


$$
\left[\bar{f}^{j}(\bar{\alpha})\right]_{\bar{f}^{\ell}}^{(m)}=q_{*}\left(\left[f^{j}(\beta)\right]_{f^{\ell}}^{(m)}\right)=[\bar{\alpha}]_{\bar{f}^{\ell}}^{(m)},
$$

so we must have $j=0$. Therefore $\left|\sigma^{-1}\left([\beta]_{f}^{(n)}\right) \cap q_{*}^{-1}\left([\bar{\alpha}]_{\bar{f}^{\ell}}^{(m)}\right)\right|=1$ for every $[\beta]_{f}^{(n)} \in q_{*}^{-1}\left([\bar{\alpha}]_{\bar{f}}^{(n)}\right)$, which proves the last statement of the lemma.

For $\ell \mid n$, we have a commutative diagram of pointed sets

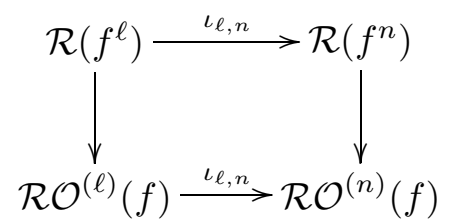

where the vertical maps are projections, and the horizontal maps are induced by the level-change function $\iota_{\ell, n}: G \rightarrow G$ defined (as in [5, Definition 1.9]) by

$$
\iota_{\ell, n}(\beta):=\beta f^{\ell}(\beta) f^{2 \ell}(\beta) \cdots f^{n-\ell}(\beta) .
$$

Definition 1.8. We say that an $f$-orbit $[\alpha]_{f}^{(n)} \in \mathcal{R O}^{(n)}(f)$ is reducible to level $h$ if there exists a $[\beta]_{f}^{(h)} \in \mathcal{R O}^{(h)}(f)$ such that $\iota_{h, n}\left([\beta]_{f}^{(h)}\right)=[\alpha]_{f}^{(n)}$. The lowest level $d=d\left([\alpha]_{f}^{(n)}\right)$ to which $[\alpha]_{f}^{(n)}$ reduces is its depth. Clearly, the length $\ell$ of the orbit $[\alpha]_{f}^{(n)}$ divides the depth $d$.

A Reidemeister orbit $[\alpha]_{f}^{(n)} \in \mathcal{R O}^{(n)}(f)$ is said to have the full depth property if its depth equals its length, i.e., $d=\ell$.

For example, $[1]_{f}^{(n)} \in \mathcal{R O}^{(n)}(f)$ always has full depth because its depth is 1. Example of nonfull depth: If $f: G \rightarrow G$ is the identity automorphism, then any orbit $[\alpha]_{f}^{(n)}$ has length $\ell=1$, but the depth $d$ varies.

Corollary 1.9. Let $n>0$ and $\alpha \in G$. Assume that the orbit $[\bar{\alpha}]_{\bar{f}}^{(n)} \in$ $\mathcal{R O}^{(n)}(\bar{f})$ is irreducible and has the full depth property. Then in the commutative diagram of exact sequences

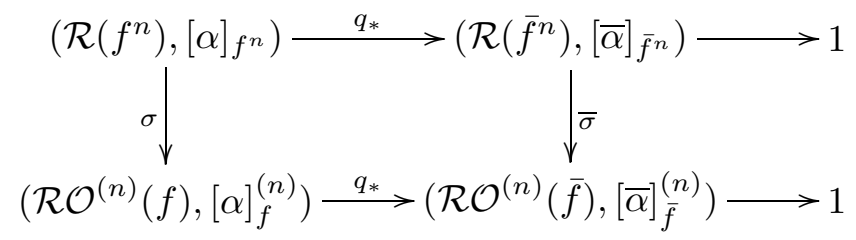

$\sigma$ restricts to a bijection

$$
\sigma: q_{*}^{-1}\left([\bar{\alpha}]_{\bar{f}^{n}}\right) \rightarrow q_{*}^{-1}\left([\bar{\alpha}]_{\bar{f}}^{(n)}\right) .
$$

Proof. If the orbit $[\bar{\alpha}]_{\bar{f}}^{(n)}$ is irreducible, then the depth of the orbit is $n$. Apply Lemma 1.7 to $\ell=d=n$. 
Notation. For $\alpha \in G$, let $\tau_{\alpha}: G \rightarrow G$ denote the conjugation defined by $\tau_{\alpha}(\beta)=\alpha \beta \alpha^{-1}$.

Lemma 1.10. For any $\alpha \in G$, there is a canonical bijection of the Reidemeister orbit sets of $\tau_{\alpha} f$ and $f$, denoted by $\alpha_{*}: \mathcal{R O}^{(n)}\left(\tau_{\alpha} f\right) \rightarrow \mathcal{R O}^{(n)}(f)$, given by

$$
\alpha_{*}\left([g]_{\tau_{\alpha} f}^{(n)}\right)=\left[g \alpha f(\alpha) \cdots f^{n-1}(\alpha)\right]_{f}^{(n)} .
$$

Proof. Clearly we have

$$
\left(\tau_{\alpha} f\right)^{n}(g)=\left(\alpha f(\alpha) \cdots f^{n-1}(\alpha)\right) f^{n}(g)\left(\alpha f(\alpha) \cdots f^{n-1}(\alpha)\right)^{-1}
$$

for all $g \in G$. By $[2$, p. 4], there is a canonical bijection

$$
\alpha_{*}: \mathcal{R}\left(\left(\tau_{\alpha} f\right)^{n}\right) \rightarrow \mathcal{R}\left(f^{n}\right)
$$

defined by $\alpha_{*}\left([g]_{\left(\tau_{\alpha} f\right)^{n}}\right)=\left[g \alpha f(\alpha) \cdots f^{n-1}(\alpha)\right]_{f^{n}}$ for all $g \in G$.

We will show that under this bijection $\alpha_{*}$, the $\tau_{\alpha} f$-action on $\mathcal{R}\left(\left(\tau_{\alpha} f\right)^{n}\right)$ corresponds to the $f$-action on $\mathcal{R}\left(f^{n}\right)$. That is to say, we show the commutativity of the following diagram:

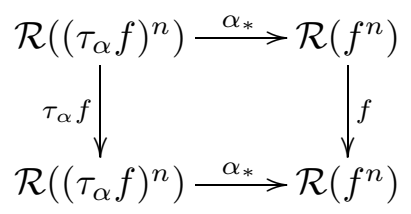

Then $\alpha_{*}$ clearly induces the desired bijection between the Reidemeister orbit sets.

Let $[g]_{\left(\tau_{\alpha} f\right)^{n}}$ be an arbitrary element in $\mathcal{R}\left(\left(\tau_{\alpha} f\right)^{n}\right)$. Then

$$
\begin{aligned}
& \alpha_{*} \tau_{\alpha} f\left([g]_{\left(\tau_{\alpha} f\right)^{n}}\right)=\alpha_{*}\left(\left[\tau_{\alpha} f(g)\right]_{\left(\tau_{\alpha} f\right)^{n}}\right)=\left[\tau_{\alpha} f(g) \alpha f(\alpha) \cdots f^{n-1}(\alpha)\right]_{f^{n}} \\
& =\left[\alpha f\left(g \alpha f(\alpha) \cdots f^{n-1}(\alpha)\right) f^{n}\left(\alpha^{-1}\right)\right]_{f^{n}} \\
& =\left[f\left(g \alpha f(\alpha) \cdots f^{n-1}(\alpha)\right)\right]_{f^{n}}=f \alpha_{*}\left([g]_{\left(\tau_{\alpha} f\right)^{n}}\right) .
\end{aligned}
$$

This is exactly what we need.

Combining Lemma 1.7 with Lemma 1.10 (applied to the endomorphism $f^{d}$ ), we have

Corollary 1.11. Let $n>0$ and $\alpha \in G$. Suppose the orbit $[\bar{\alpha}]_{\bar{f}}^{(n)} \in$ $\mathcal{R O}^{(n)}(\bar{f})$ has depth $d$, and let $m:=n / d$ and $\bar{\iota}_{d, n}(\bar{\beta})=\bar{\alpha}$ for some $\beta \in G$. Then we have a commutative diagram of exact sequences in the category of pointed sets: 


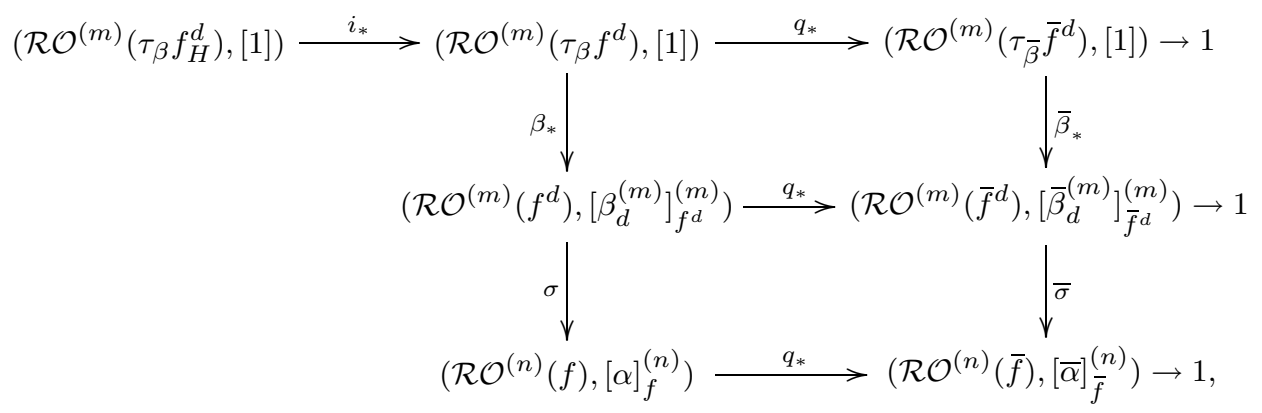

where the vertical maps $\beta_{*}$ and $\bar{\beta}_{*}$ are induced by the element $\beta \in G$. The notation [1] in the middle of the upper sequence stands for $[1]_{\tau_{\beta} f^{d}}^{(m)}$, and the other ocurrences of [1] have a similar meaning. The notation $\beta_{d}^{(m)}$ stands for $\beta f^{d}(\beta) \cdots f^{n-d}(\beta)$, and similarly for $\bar{\beta}_{d}^{(m)}$. Furthermore, if $[\bar{\alpha}]_{\bar{f}}^{(n)}$ has the full depth property and $\bar{\iota}_{d, n}(\bar{\beta})=\bar{\alpha}$, then we have a bijection

$$
\sigma \circ \beta_{*}: q_{*}^{-1}\left([1]_{\tau_{\bar{\beta}} \bar{f}^{d}}^{(m)}\right) \rightarrow q_{*}^{-1}\left([\bar{\alpha}]_{\bar{f}}^{(n)}\right) .
$$

Proof. In the notation of Lemma 1.10, the canonical bijection $\beta_{*}$ is given by $\beta_{*}\left([g]_{\tau_{\beta} f^{d}}^{(m)}\right)=\left[g \beta f^{d}(\beta) \cdots f^{n-d}(\beta)\right]_{f^{d}}^{(m)}$. Then we have

$$
\begin{aligned}
\bar{\sigma} \circ \bar{\beta}_{*} \circ q_{*}\left([1]_{\tau_{\beta} f^{d}}^{(m)}\right) & =\bar{\sigma} \circ \bar{\beta}_{*}\left([1]_{\tau_{\bar{\beta}} \bar{f}^{d}}^{(m)}\right)=\bar{\sigma}\left(\left[\bar{\beta} \bar{f}^{d}(\bar{\beta}) \cdots \bar{f}^{n-d}(\bar{\beta})\right]_{\bar{f}^{d}}^{(m)}\right) \\
& =\left[\bar{\beta} \bar{f}^{d}(\bar{\beta}) \cdots \bar{f}^{n-d}(\bar{\beta})\right]_{\bar{f}}^{(n)}=\left[\bar{\iota}_{d, n}(\bar{\beta})\right]_{\bar{f}}^{(n)}=[\bar{\alpha}]_{\bar{f}}^{(n)} .
\end{aligned}
$$

When $[\bar{\alpha}]_{\bar{f}}^{(n)}$ has the full depth property, the depth $d$ is the same as the length $\ell$. Hence the last statement follows by Lemma 1.7.

Lemma 1.12. $\operatorname{Fix}\left(\tau_{\alpha} f^{n}\right)=\{1\}$ implies the full depth property of $[\alpha]_{f}^{(n)}$.

Proof. Let $d$ and $\ell$ denote the depth and length of $[\alpha]_{f}^{(n)}$, respectively. Clearly $\ell$ divides $d$.

Since $\alpha$ and $f^{\ell}(\alpha)$ are in the same Reidemeister class of $f^{n}$, there is an $\omega \in G$ such that $\alpha=\omega f^{\ell}(\alpha) f^{n}\left(\omega^{-1}\right)$. Let $\mu:=\omega f^{\ell}(\omega) \cdots f^{n-\ell}(\omega)$. Then

$$
\begin{aligned}
\alpha & =\omega f^{\ell}(\alpha) f^{n}\left(\omega^{-1}\right) \\
& =\omega f^{\ell}\left(\omega f^{\ell}(\alpha) f^{n}\left(\omega^{-1}\right)\right) f^{n}\left(\omega^{-1}\right)=\omega f^{\ell}(\omega) f^{2 \ell}(\alpha) f^{n}\left(f^{\ell}\left(\omega^{-1}\right) \omega^{-1}\right) \\
& =\cdots \\
& =\mu f^{n}(\alpha) f^{n}\left(\mu^{-1}\right)=\left(\mu \alpha^{-1}\right) \alpha f^{n}\left(\alpha \mu^{-1}\right),
\end{aligned}
$$

and $\alpha \mu^{-1} \in \operatorname{Fix}\left(\tau_{\alpha} f^{n}\right)$.

When $\operatorname{Fix}\left(\tau_{\alpha} f^{n}\right)=\{1\}$, we have $\alpha=\mu=\omega f^{\ell}(\omega) \cdots f^{n-\ell}(\omega)$. By definition this means $[\alpha]_{f}^{(n)}$ is reducible to level $\ell$. Therefore $d \leq \ell$ by the definition of depth. But $\ell \mid d$, so $\ell=d$. 
Note that the converse of Lemma 1.12 does not hold. If we let $f: G \rightarrow G$ be the identity map and $\alpha=1 \in G$, then the orbit $[\alpha]_{f}^{(n)}$ has the full depth property, but $\operatorname{Fix}\left(\tau_{\alpha} f^{n}\right)=G$.

Corollary 1.13. Suppose $\bar{\iota}_{d, n}(\bar{\beta})=\bar{\alpha}$. Then $\left(\tau_{\bar{\beta}} \bar{f}^{d}\right)^{m}=\tau_{\bar{\alpha}} \bar{f}^{n}$. If $\operatorname{Fix}\left(\tau_{\bar{\alpha}} \bar{f}^{n}\right)=\{1\}$, then

$$
i_{*}: \mathcal{R} \mathcal{O}^{(m)}\left(\tau_{\beta} f_{H}^{d}\right) \rightarrow q_{*}^{-1}\left([\bar{\alpha}]_{\bar{f}}^{(n)}\right) \subset \mathcal{R} \mathcal{O}^{(n)}(f)
$$

is a bijection.

Proof. By direct computation, $\left(\tau_{\bar{\beta}} \bar{f}^{d}\right)^{m}=\tau_{\overline{\iota_{d, n}}(\bar{\beta})} \bar{f}^{n}=\tau_{\bar{\alpha}} \bar{f}^{n}$.

When $\operatorname{Fix}\left(\tau_{\bar{\alpha}} \bar{f}^{n}\right)=\{1\}$, Lemma 1.12 tells us that $[\bar{\alpha}]_{\bar{f}}^{(n)}$ has the full depth property. Thus by Corollary 1.11 we have a bijection

$$
\sigma \circ \beta_{*}: q_{*}^{-1}\left([1]_{\tau_{\bar{\beta}} \bar{f}^{d}}^{(m)}\right) \rightarrow q_{*}^{-1}\left([\bar{\alpha}]_{\bar{f}}^{(n)}\right) .
$$

On the other hand, since $\operatorname{Fix}\left(\left(\tau_{\bar{\beta}} \bar{f}^{d}\right)^{m}\right)=\{1\}$, by Corollary 1.6 the map

$$
i_{*}: \mathcal{R} \mathcal{O}^{(m)}\left(\tau_{\beta} f_{H}^{d}\right) \rightarrow q_{*}^{-1}\left([1]_{\tau_{\bar{\beta}} \bar{f}^{d}}^{(m)}\right) \subset \mathcal{R} \mathcal{O}^{(m)}\left(\tau_{\beta} f^{d}\right)
$$

is a bijection. Combining the two bijections, we have the assertion.

Theorem 1.14. Let $n>0$. For an orbit $[\bar{\alpha}]_{\bar{f}}^{(n)} \in \mathcal{R O}^{(n)}(\bar{f})$, let $d_{\alpha}$ be the depth of $[\bar{\alpha}]_{\bar{f}}^{(n)}, m_{\alpha}:=n / d_{\alpha}$, and $\bar{\iota}_{d_{\alpha}, n}\left(\bar{\beta}_{\alpha}\right)=\bar{\alpha}$ for some $\beta_{\alpha} \in G$. If $\operatorname{Fix}\left(\tau_{\bar{\alpha}} \bar{f}^{n}\right)=\{1\}$ for all $[\bar{\alpha}]_{\bar{f}}^{(n)} \in \mathcal{R O}^{(n)}(\bar{f})$, then

$$
\sharp \mathcal{R} \mathcal{O}^{(n)}(f)=\sum_{[\bar{\alpha}]_{\bar{f}}^{(n)} \in \mathcal{R} \mathcal{O}^{(n)}(\bar{f})} \sharp \mathcal{R} \mathcal{O}^{\left(m_{\alpha}\right)}\left(\tau_{\beta_{\alpha}} f_{H}^{d_{\alpha}}\right) .
$$

Proof. Clearly the Reidemeister orbit set $\mathcal{R O}^{(n)}(f)$ is the disjoint union of $q_{*}^{-1}\left([\bar{\alpha}]_{\bar{f}}^{(n)}\right)$ for all $[\bar{\alpha}]_{\bar{f}}^{(n)} \in \mathcal{R} \mathcal{O}^{(n)}(\bar{f})$. By Corollary 1.13,

$$
i_{*}: \mathcal{R} \mathcal{O}^{\left(m_{\alpha}\right)}\left(\tau_{\beta_{\alpha}} f_{H}^{d_{\alpha}}\right) \rightarrow q_{*}^{-1}\left([\bar{\alpha}]_{\bar{f}}^{(n)}\right) \subset \mathcal{R} \mathcal{O}^{(n)}(f)
$$

is a bijection for every $[\bar{\alpha}]_{\bar{f}}^{(n)} \in \mathcal{R} \mathcal{O}^{(n)}(\bar{f})$. This completes the proof.

EXAMPLE 1.15 (Semidirect product of finitely generated free abelian groups). Let $H=\mathbb{Z}^{r}$ and $\bar{G}=\mathbb{Z}^{k}$ be two (additive) finitely generated free abelian groups and let $M: \bar{G} \rightarrow \operatorname{Aut}(H)$ be a homomorphism from $\bar{G}$ to the automorphism group of $H$. Denote by $M_{\bar{g}}:=M(\bar{g})$ the image of each $\bar{g} \in \bar{G}$. Let $G$ be the external semidirect product of $H$ and $\bar{G}$ via $M$; it is the set of all pairs $(\bar{g}, h) \in \bar{G} \times H$ with the group operation $\left(\bar{g}_{1}, h_{1}\right)+\left(\bar{g}_{2}, h_{2}\right)=$ $\left(\bar{g}_{1}+\bar{g}_{2}, M_{\bar{g}_{2}}\left(h_{1}\right)+h_{2}\right)$. Let $f: G \rightarrow G$ be an endomorphism such that $f(H) \subset H$. Then $\bar{f}: \bar{G} \rightarrow \bar{G}$ and $f_{H}: H \rightarrow H$ are defined by two matrices 
$\bar{F} \in \operatorname{Mat}_{k, k}(\mathbb{Z})$ and $F_{H} \in \operatorname{Mat}_{r, r}(\mathbb{Z})$ (see [2, Example 3]). Since the sequence

$$
0 \rightarrow \operatorname{Fix}\left(\bar{f}^{n}\right) \rightarrow \bar{G} \stackrel{1-\bar{f}^{n}}{\longrightarrow} \bar{G} \rightarrow \bar{G} / \operatorname{Im}\left(1-\bar{f}^{n}\right) \rightarrow 0
$$

is exact, $\operatorname{Fix}\left(\bar{f}^{n}\right) \neq\{0\}$ if and only if $\operatorname{det}\left(I-\bar{F}^{n}\right)=0$, and this happens if and only if $\sharp \mathcal{R} \mathcal{O}^{(n)}(\bar{f})=\infty$. Therefore either $\mathcal{R \mathcal { O } ^ { ( n ) }}(f)$ and $\mathcal{R O}^{(n)}(\bar{f})$ are infinite, or $\operatorname{Fix}\left(\bar{f}^{n}\right)=\{0\}$. In this last case, since $\bar{G}$ is abelian, Theorem 1.14 can be used; thus

$$
\sharp \mathcal{R} \mathcal{O}^{(n)}(f)=\sum_{[\bar{\alpha}]_{\bar{f}}^{(n)} \in \mathcal{R} \mathcal{O}^{(n)}(\bar{f})} \sharp \mathcal{R} \mathcal{O}^{\left(m_{\alpha}\right)}\left(f_{H}^{d_{\alpha}}\right) .
$$

EXAMPLE 1.16 (The Klein bottle). Let $G$ be the fundamental group of the Klein bottle, i.e., $G:=\left\langle\alpha, \beta \mid \beta \alpha=\alpha^{-1} \beta\right\rangle$. The subgroup $H:=\langle\alpha\rangle$ is a fully invariant normal subgroup of $G$ and if $M: \mathbb{Z} \rightarrow \operatorname{Aut}(\mathbb{Z})=\{1,-1\}$ is the homomorphism defined by $M(k)=(-1)^{k}$ for all $k \in \mathbb{Z}$ then $G$ is the semidirect product of $H$ and $\bar{G}:=\mathbb{Z}$ via $M$. So let $f: G \rightarrow G$ be an endomorphism. Then $f_{H}: H \rightarrow H$ and $\bar{f}: G / H \cong \bar{G} \rightarrow \bar{G}$ are defined by elements of $\operatorname{Mat}_{1,1}(\mathbb{Z})$, i.e. by integers $u$ and $w$. In other words, $f_{H}(a)=u a$ for all $a \in H$ and $\bar{f}(b)=w b$ for all $b \in G / H \cong \bar{G}$ (see [2, Example 4]).

We will calculate $\mathcal{R O}^{(2)}(f)$ when $u=2$ and $w=3$. Using the exact sequence in the previous example for $n=2$, we can identify the 2-periodic point classes of $\bar{f}$, i.e., the elements of $\mathcal{R}\left(\bar{f}^{2}\right)$, with the elements of $\mathbb{Z}_{8}$. Thus

$$
\mathcal{R} \mathcal{O}^{(2)}(\bar{f})=\left\{[0]_{\bar{f}}^{(2)},[1]_{\bar{f}}^{(2)},[2]_{\bar{f}}^{(2)},[4]_{\bar{f}}^{(2)},[5]_{\bar{f}}^{(2)}\right\}
$$

where $[0]_{\bar{f}}^{(2)}=\left\{[0]_{\bar{f}^{2}}\right\},[1]_{\bar{f}}^{(2)}=\left\{[1]_{\bar{f}^{2}},[3]_{\bar{f}^{2}}\right\},[2]_{\bar{f}}^{(2)}=\left\{[2]_{\bar{f}^{2}},[6]_{\bar{f}^{2}}\right\},[4]_{\bar{f}}^{(2)}=$ $\left\{[4]_{\bar{f}^{2}}\right\}$ and $[5]_{\bar{f}}^{(2)}=\left\{[5]_{\bar{f}^{2}},[7]_{\bar{f}^{2}}\right\}$. For period 1, the set $\mathcal{R} \mathcal{O}^{(1)}(\bar{f})=\mathcal{R}(\bar{f}) \cong$ $\mathbb{Z}_{2}$ is $\left\{[0]_{\bar{f}},[1]_{\bar{f}}\right\}$. Since $\bar{\iota}_{1,2}$ is multiplication by $1+3=4$, the $\bar{f}$-orbits $[0]_{\bar{f}}^{(2)}$ and $[4]_{\bar{f}}^{(2)}$ are reducible to 1 , the others are irreducible. Thus any orbit of $\mathcal{R O}^{(2)}(\bar{f})$ has the full depth property, and by the previous example, we have

$$
\sharp \mathcal{R} \mathcal{O}^{(2)}(f)=2 \sharp \mathcal{R} \mathcal{O}^{(2)}\left(f_{H}\right)+3 \sharp \mathcal{R}\left(f_{H}^{2}\right) .
$$

Furthermore, the set $\mathcal{R} \mathcal{O}^{(2)}\left(f_{H}\right)$ is $\left\{[0]_{f_{H}}^{(2)},[1]_{f_{H}}^{(2)}\right\}$, where $[0]_{f_{H}}^{(2)}=\left\{[0]_{f_{H}^{2}}\right\}$ and $[1]_{f_{H}}^{(2)}=\left\{[1]_{f_{H}^{2}},[2]_{f_{H}^{2}}\right\}$; and the set $\mathcal{R}\left(f_{H}^{2}\right) \cong \mathbb{Z}_{3}$ is $\left\{[0]_{f_{H}^{2}},[1]_{f_{H}^{2}},[2]_{f_{H}^{2}}\right\}$. Therefore

$$
\sharp \mathcal{R} \mathcal{O}^{(2)}(f)=13 \text {. }
$$

2. Nielsen type essential $n$-orbit numbers. Let $X$ be a compact connected ANR. Let $f: X \rightarrow X$ be a map. The set of fixed point classes will be denoted by $\mathcal{F P}(f)$. 
Let $n>0$ be a given integer. Fixed point classes of the iterate $f^{n}: X \rightarrow$ $X$ will also be called $n$-periodic point classes of $f$. Then $f$ acts on the set $\mathcal{F P}\left(f^{n}\right)$ by $\boldsymbol{A}_{f^{n}} \mapsto f\left(\boldsymbol{A}_{f^{n}}\right)$. The $f$-orbit of a class $\boldsymbol{A}_{f^{n}}$ will be called an $n$-orbit class, denoted by $\boldsymbol{A}_{f}^{(n)}$. The set of $n$-orbit classes will be denoted by $\mathcal{O}^{(n)}(f)$.

The length of the orbit $\boldsymbol{A}_{f}^{(n)}$ is the smallest integer $\ell>0$ such that $\boldsymbol{A}_{f^{n}}=f^{\ell}\left(\boldsymbol{A}_{f^{n}}\right)$. Clearly $\ell$ divides $n$ because $\boldsymbol{A}_{f^{n}}=f^{n}\left(\boldsymbol{A}_{f^{n}}\right)$.

Standard fixed point index theory provides an integer index ind $\left(\boldsymbol{A}_{f^{n}}\right)$ for each periodic point class $\boldsymbol{A}_{f^{n}}$. A periodic point class $\boldsymbol{A}_{f^{n}}$ is essential if its index is nonzero. We let $\mathcal{E}\left(f^{n}\right)$ be the set of essential periodic point classes of $f$. Then $N\left(f^{n}\right)$, the Nielsen number of $f^{n}$, is the cardinality of $\mathcal{E}\left(f^{n}\right)$.

Definition 2.1. Let $n>0$ be a given integer. Then $f$ acts on $\mathcal{E}\left(f^{n}\right)$ by $\boldsymbol{F}_{f^{n}} \mapsto f\left(\boldsymbol{F}_{f^{n}}\right)$. The $f$-orbit of an essential fixed point class $\boldsymbol{F}_{f^{n}}$ of $f^{n}$ will be called an essential n-orbit class, denoted by $\boldsymbol{F}_{f}^{(n)}$. Since $\operatorname{ind}\left(f\left(\boldsymbol{F}_{f^{n}}\right)\right)=$ $\operatorname{ind}\left(\boldsymbol{F}_{f^{n}}\right)$, the index $\operatorname{ind}\left(\boldsymbol{F}_{f}^{(n)}\right)$ of an essential $n$-orbit class equals

$$
\ell\left(\boldsymbol{F}_{f}^{(n)}\right) \cdot \operatorname{ind}\left(\boldsymbol{F}_{f^{n}}\right),
$$

where $\ell\left(\boldsymbol{F}_{f}^{(n)}\right)$ is the length of $\boldsymbol{F}_{f}^{(n)}$. The set of essential $n$-orbit classes will be denoted by $\mathcal{E O}^{(n)}(f)$.

We define the essential n-orbit number $E O^{(n)}(f)$ to be the cardinality of the set $\mathcal{E} \mathcal{O}^{(n)}(f)$. This number is a homotopy invariant (see [6, III.3.3, III.3.4]). It is a Nielsen type number in the general sense of [6, III.4.8].

Let $x_{0}$ be the base point in $X$, and take a path $w$ from $x_{0}$ to $f\left(x_{0}\right)$ as the base path for $f$. The induced endomorphism $f_{*}^{x_{0}}: \pi_{1}\left(X, x_{0}\right) \rightarrow \pi_{1}\left(X, x_{0}\right)$ is defined by

$$
f_{*}^{x_{0}}(\langle\gamma\rangle):=\left\langle w f(\gamma) w^{-1}\right\rangle \quad \text { for any loop } \gamma \text { at } x_{0} .
$$

Note that although the base path $w$ is not shown in the notation, the endomorphism $f_{*}^{x_{0}}$ depends on the homotopy class of $w$. For $n>1,\left(f^{n}\right)_{*}^{x_{0}}=$ $\left(f_{*}^{x_{0}}\right)^{n}$ if the base path for $f^{n}$ is taken to be $w f(w) \cdots f^{n-1}(w)$.

It is well known that every fixed point class of $f$ is assigned a Reidemeister class in $\mathcal{R}\left(f_{*}^{x_{0}}\right)$, called its coordinate. We get an injection

$$
\rho: \mathcal{F P}(f) \hookrightarrow \mathcal{R}\left(f_{*}^{x_{0}}\right),
$$

where $\mathcal{R}\left(f_{*}^{x_{0}}\right)$ is the Reidemeister set in $\pi_{1}\left(X, x_{0}\right)$ as in Section 1 , defined by

$$
\rho\left(\boldsymbol{A}_{f}\right):=\left[c f\left(c^{-1}\right) w^{-1}\right]_{f_{*}}^{x_{0}}
$$

for any path $c$ from $x_{0}$ to a point $x$ in $\boldsymbol{A}_{f}$. Thus we also get an injection

$$
\rho: \mathcal{O}^{(n)}(f) \hookrightarrow \mathcal{R} \mathcal{O}^{(n)}\left(f_{*}^{x_{0}}\right),
$$


defined by

$$
\rho\left(\boldsymbol{A}_{f}^{(n)}\right):=\left[c f^{n}\left(c^{-1}\right) f^{n-1}\left(w^{-1}\right) \cdots f\left(w^{-1}\right) w^{-1}\right]_{f_{*}^{x_{0}}}^{(n)}
$$

for any path $c$ from $x_{0}$ to a point $x$ in $\boldsymbol{A}_{f}^{(n)}$. If $\ell \mid n$ and an $\ell$-orbit class $\boldsymbol{B}_{f}^{(\ell)}$ lies inside an $n$-orbit class $\boldsymbol{A}_{f}^{(n)}$, then their coordinates are related by

$$
\rho\left(\boldsymbol{A}_{f}^{(n)}\right)=\iota_{\ell, n}\left(\rho\left(\boldsymbol{B}_{f}^{(\ell)}\right)\right),
$$

where $\iota_{\ell, n}$ is the level-change function of Section 1 , hence $\rho\left(\boldsymbol{A}_{f}^{(n)}\right)$ is reducible to level $\ell$. The depth of an $n$-orbit class $\boldsymbol{A}_{f}^{(n)}$ is defined to be the depth of its coordinate $\rho\left(\boldsymbol{A}_{f}^{(n)}\right)$.

Recall the effect of a homotopy. For a homotopy $H=\left\{h_{t}: X \rightarrow X\right\}_{0<t<1}$ connecting $f=h_{0}$ and $g=h_{1}$, we have $g_{*}^{x_{0}}=f_{*}^{x_{0}}$ if the base path for $g$ is taken to be $w H\left(x_{0}\right)$ (i.e. $w$ followed by the trace of $H$ ), where $H\left(x_{0}\right)$ stands for the path $\left\{h_{t}\left(x_{0}\right)\right\}_{0 \leq t \leq 1}$. Now an $n$-periodic point class $\boldsymbol{A}_{f^{n}}$ of $f$ corresponds to an $n$-periodic point class $\boldsymbol{B}_{g^{n}}$ of $g$ under the homotopy $H$ if and only if $\rho\left(\boldsymbol{A}_{f^{n}}\right)=\rho\left(\boldsymbol{B}_{g^{n}}\right)$ in the (same) set $\mathcal{R}\left(\left(f_{*}^{x_{0}}\right)^{n}\right)=\mathcal{R}\left(\left(g_{*}^{x_{0}}\right)^{n}\right)$.

REDUCING Lemma 2.2. Let $X$ be a compact connected ANR, and $f$ : $X \rightarrow X$ be a map. Suppose $x \in \operatorname{Fix}\left(f^{n}\right)$ lies in an $n$-orbit class $\boldsymbol{A}_{f}^{(n)}$ of depth $d$. Then there exists a homotopy $H=\left\{h_{t}: X \rightarrow X\right\}_{0 \leq t \leq 1}$ connecting $f=h_{0}$ and $g=h_{1}$ such that

(1) $x \in \operatorname{Fix}\left(g^{d}\right)$.

(2) The loop $H^{n}(x)=\left\{h_{t}^{n}(x)\right\}_{0 \leq t \leq 1}$ is contractible in $X$.

(3) $H$ equals $f$ outside an arbitrarily given neighbourhood of the point $f^{d-1}(x)$.

Note that in (2) the notation $H^{n}=\left\{h_{t}^{n}: X \rightarrow X\right\}$ stands for the homotopy from $f^{n}=h_{0}^{n}$ to $g^{n}=h_{1}^{n}$ consisting of $h_{t}^{n}$, the $n$-fold iterate of $h_{t}: X \rightarrow X$. The loop $H^{n}(x)=\left\{h_{t}^{n}(x)\right\}_{0 \leq t \leq 1}$ is the trace of the point $x$ under this homotopy.

Proof. Take the point $x$ as the base point, and take a path $w$ from $x$ to $f(x)$ as the base path for $f$. Denote the induced endomorphism $f_{*}^{x}$ : $\pi_{1}(X, x) \rightarrow \pi_{1}(X, x)$ by $\varphi$. For any $k>0$, using $w_{k}:=w f(w) \cdots f^{k-1}(w)$ as the base path for the iterate $f^{k}$, we have $\left(f^{k}\right)_{*}^{x}=\left(f_{*}^{x}\right)^{k}=\varphi^{k}$.

The $n$-periodic point class $\boldsymbol{A}_{f^{n}}$ containing $x$ has coordinate $\rho\left(\boldsymbol{A}_{f^{n}}\right)=$ $\left[\left\langle w_{n}^{-1}\right\rangle\right]_{\varphi^{n}}$ (by using the constant path at $x$ as the path $c$ in the definition of $\rho$ ).

The depth assumption means that there exists a loop $\beta$ at $x$ such that

$$
\left\langle w_{n}^{-1}\right\rangle=\langle\beta\rangle \varphi^{d}(\langle\beta\rangle) \cdots \varphi^{n-d}(\langle\beta\rangle) .
$$

Let $u$ be the path $\beta w_{d}$ from $x$ to $f^{d}(x)$. 
The depth assumption ensures that the points $\left\{f^{i}(x) \mid 0 \leq i<d\right\}$ are all different. (If not, $f^{i}(x)=f^{j}(x)$ for some $0 \leq i<j<d$. Then $x=f^{n}(x)=f^{n-i} f^{i}(x)=f^{n-i} f^{j}(x)=f^{j-i} f^{n}(x)=f^{j-i}(x)$. So $x=f^{k}(x)$ where $k=\operatorname{gcd}(n, j-i)$. Now that $\boldsymbol{A}_{f^{n}}$ contains a $k$-periodic point $x$, its coordinate $\rho\left(\boldsymbol{A}_{f^{n}}\right)$ would be reducible to the level $k$ which is smaller than the depth $d$. This contradicts the definition of depth.)

By the homotopy extension property of ANR spaces, there exists a homotopy $H=\left\{h_{t}: X \rightarrow X\right\}_{0 \leq t \leq 1}$, differing from $f$ only in a small neighbourhood of the point $f^{d-1}(x)$, such that $h_{0}=f$ and $h_{t}^{d}(x)=u(1-t)$ for all $t$. Let $g=h_{1}$. Clearly $g^{d}(x)=u(0)=x$.

It remains to verify (2). The trace of the homotopy $H^{d}=\left\{h_{t}^{d}: X \rightarrow X\right\}$ is $H^{d}(x)=u^{-1}$. Let $m:=n / d$; then $H^{n}=\left(H^{d}\right)^{m}$. We shall construct a homotopy that contracts the trace $H^{n}(x)$ to a point. The symbol $\simeq$ means homotopy between paths, relative to end points. Note that two paths can be multiplied only under conditions on their end points, and the product of paths is not commutative. Since on the cube $I^{m}$ the diagonal is homotopic to a product of $m$ edges, we have

$$
\begin{array}{rlr}
H^{n}(x) & =\left\{\left(h_{t}^{d}\right)^{m}(x)\right\}_{t \in I} \simeq \prod_{i=m-1}^{0}\left\{f^{i d} h_{t}^{d} g^{(m-i-1) d}(x)\right\}_{t \in I} \\
& =\prod_{i=m-1}^{0} f^{i d}\left(H^{d}(x)\right) & \text { since } g^{d}(x)=x \\
& \simeq \prod_{i=m-1}^{0} f^{i d}\left(w_{d}^{-1} \beta^{-1}\right) & \text { since } H^{d}(x)=u^{-1} \simeq w_{d}^{-1} \beta^{-1} \\
& \simeq \prod_{i=m-1}^{0}\left(w_{(i+1) d}^{-1} w_{i d} f^{i d}\left(\beta^{-1}\right)\right) & \text { since } w_{(i+1) d}=w_{i d} f^{i d}\left(w_{d}\right) \\
& =w_{n}^{-1} \prod_{i=m-1}^{0}\left(w_{i d} f^{i d}\left(\beta^{-1}\right) w_{i d}^{-1}\right) .
\end{array}
$$

Hence

$$
\left\langle H^{n}(x)\right\rangle=\left\langle w_{n}^{-1}\right\rangle \prod_{i=m-1}^{0}\left(f^{i d}\right)_{*}^{x}\left(\left\langle\beta^{-1}\right\rangle\right)=\left\langle w_{n}^{-1}\right\rangle \prod_{i=m-1}^{0} \varphi^{i d}\left(\left\langle\beta^{-1}\right\rangle\right)=1 .
$$

This completes the proof of the lemma.

We will need the mod $K$ version of the Nielsen theory. If $f, X$ and $x$ are as above, and if $K$ is an $f_{*}^{x}$-invariant normal subgroup of $\pi_{1}(X, x)$, then we denote the induced homomorphism on $\pi_{1}(X, x) / K$ by $f_{* / K}^{x}$. We then have the set $\mathcal{R} \mathcal{O}^{(n)}\left(f_{* / K}^{x}\right)$ of Reidemeister $f_{* / K}^{x}$-orbits, and the mod $K$ essential 
$n$-orbit number $E O_{K}^{(n)}(f)$, that is, the cardinality of the set $\mathcal{E} \mathcal{O}_{K}^{(n)}(f)$ of $\bmod$ $K$ essential $n$-orbit classes. We also have an injection

$$
\rho_{K}: \mathcal{O}_{K}^{(n)}(f) \hookrightarrow \mathcal{R} \mathcal{O}^{(n)}\left(f_{* / K}^{x}\right) .
$$

We will omit the subscript $K$ when the normal subgroup $K$ is clear from the context.

In this paper we will assume that all of our fibrations $F \hookrightarrow E \rightarrow B$ (with projection $p: E \rightarrow B$ ) are Hurewicz fibrations with path connected fibres (see [7]). We say that $f: E \rightarrow E$ is a fibre preserving map provided there is a well defined map $\bar{f}: B \rightarrow B$ with $p f=\bar{f} p$. When such a map exists it is unique, and when $B$ is a path connected locally path connected space it is enough that for all $b \in B$ the restriction of $f$ takes the fibre $F_{b}:=p^{-1}(b)$ to another fibre. We will refer to the pair $(f, \bar{f})$ as a fibre preserving map. If $(f, \bar{f})$ is a fibre preserving map, then for any $b \in \operatorname{Fix}\left(\bar{f}^{n}\right)$ we will denote the restricted map on $F_{b}$ by $f_{b}^{n}$. For $x \in E$ let $j: F_{p(x)} \rightarrow E$ be the inclusion and let $K$ denote the kernel of the homomorphism

$$
j_{*}: \pi_{1}\left(F_{p(x)}, x\right) \rightarrow \pi_{1}(E, x) .
$$

Proposition 2.3. Let $p: E \rightarrow B$ be a fibration of compact connected ANR's with path connected fibres, and let $f: E \rightarrow E$ be a fibre preserving map. If $x \in E$ is in an essential n-orbit class $\boldsymbol{F}_{f}^{(n)}$ of $f$, and $p(x)$ is a fixed point of $\bar{f}^{\ell}$, where $\ell \mid n$, then the sequence (with $m:=n / \ell$ )

$$
\left(\mathcal{E} \mathcal{O}_{K}^{(m)}\left(f_{p(x)}^{\ell}\right),{ }_{K} \boldsymbol{F}_{f_{p(x)}^{\ell}}^{(m)}\right) \stackrel{j_{\mathcal{E}}}{\longrightarrow}\left(\mathcal{E} \mathcal{O}^{(m)}\left(f^{\ell}\right), \boldsymbol{F}_{f^{\ell}}^{(m)}\right) \stackrel{p_{\mathcal{E}}}{\longrightarrow}\left(\mathcal{E} \mathcal{O}^{(m)}\left(\bar{f}^{\ell}\right), \boldsymbol{F}_{\bar{f}^{\ell}}^{(m)}\right)
$$

is an exact sequence of pointed sets, where $j_{\mathcal{E}}$ and $p_{\mathcal{E}}$ are induced by the inclusion $j: F_{p(x)} \rightarrow E$ and the projection $p: E \rightarrow B$ respectively, and the base points are the essential orbit classes containing either $x$ or $p(x)$.

Note that $f_{p(x)}^{\ell}$ means the map $\left(f^{\ell}\right)_{p(x)}: F_{p(x)} \rightarrow F_{p(x)}$, because $\left(f_{p(x)}\right)^{\ell}$ does not make sense.

Proof. If $x$ is in an essential $n$-orbit class of $f$, then $x$ is in an essential $m$-orbit class of $f^{\ell}$. Applying [4, Theorem 1.1] to $f^{n}=\left(f^{\ell}\right)^{m}$, we have a commutative diagram of pointed sets

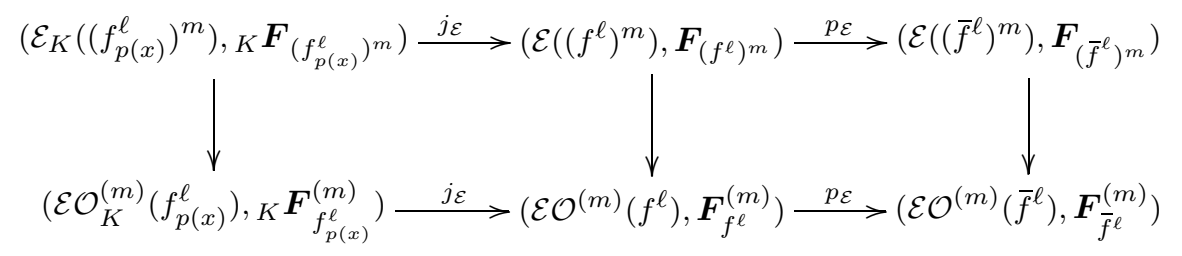

where the vertical maps are projections and the upper sequence is exact. Thus the proposition follows from the exactness of the upper sequence. Note that $p_{\mathcal{E}}$ is not necessarily surjective. 
We call a subset $\xi \subset \operatorname{Fix}\left(\bar{f}^{n}\right)$ a set of essential n-orbit representatives for $\bar{f}$ if $\xi$ contains exactly one point from each essential $n$-orbit class $\boldsymbol{F}_{\bar{f}}^{(n)} \in$ $\mathcal{E} \mathcal{O}^{(n)}(\bar{f})$.

For each $b \in \xi$, let $d$ be the depth of the essential $\bar{f}$-orbit class $\boldsymbol{F}_{\bar{f}}^{(n)}$ containing $b$. Now $b$ and $\bar{f}^{d}(b)$ are in the same fixed point class of $\bar{f}^{n}$ (because the depth is always a multiple of the length, of the $\bar{f}$-orbit class $\boldsymbol{F}_{\bar{f}}^{(n)}$ ), but not necessarily $\bar{f}^{d}(b)=b$. By the Reducing Lemma, there exists a homotopy $\bar{H}=\left\{\bar{h}_{t}: B \rightarrow B\right\}_{t \in I}$ connecting $\bar{f}=\bar{h}_{0}$ to some $\bar{g}=\bar{h}_{1}$ such that $b \in \operatorname{Fix}\left(\bar{g}^{d}\right)$, and the $n$-orbit class of $\bar{f}$ containing $b$ corresponds to the $n$-orbit class of $\bar{g}$ containing $b$ because the trace $H^{n}(x)$ is a contractible loop. We can do this for all $b \in \xi$ simultaneously, because the $\bar{H}$ above only changes $\bar{f}$ in a small neighborhood of the $\bar{f}$-orbit of $b$.

By the homotopy lifting property of the fibration $p$, the homotopy $\bar{H}$ in $B$ lifts to a fibre preserving homotopy $H=\left\{h_{t}: E \rightarrow E\right\}_{t \in I}$ connecting $f=h_{0}$ to some $g=h_{1}$.

TheOREM 2.4. Suppose $p: E \rightarrow B$ is a fibration of compact connected ANR's with path connected fibres, and $f: E \rightarrow E$ is a fibre preserving map. Let $\xi=\left\{b_{1}, \ldots, b_{k}\right\}$ be a set of essential n-orbit representatives for $\bar{f}$. If $\operatorname{Fix}\left(\left(\bar{f}^{n}\right)_{*}^{b_{i}}\right)=\{1\}$ for every $b_{i} \in \xi$, then

$$
E O^{(n)}(f)=\sum_{b_{i} \in \xi} E O_{K}^{\left(m_{i}\right)}\left(g_{b_{i}}^{d_{i}}\right),
$$

where $g$ is the fibre preserving map from the Reducing Lemma, $K$ is the kernel of the homomorphism $j_{*}: \pi_{1}\left(F_{b_{i}}\right) \rightarrow \pi_{1}(E)$ induced by the inclusion of the fibre, $d_{i}$ is the depth of the n-orbit class of $\bar{f}$ containing $b_{i}$, and $m_{i}=n / d_{i}$. In the condition $\operatorname{Fix}\left(\left(\bar{f}^{n}\right)_{*}^{b_{i}}\right)=\{1\}$, the endomorphism $\left(\bar{f}^{n}\right)_{*}^{b_{i}}: \pi_{1}\left(B, b_{i}\right) \rightarrow \pi_{1}\left(B, b_{i}\right)$ is meant to have the constant path at $b_{i}$ as base path.

Note that when $b_{i} \in \operatorname{Fix}\left(\bar{f}^{d_{i}}\right)$, the term $E O_{K}^{\left(m_{i}\right)}\left(g_{b_{i}}^{d_{i}}\right)$ in the summation can be replaced by $E O_{K}^{\left(m_{i}\right)}\left(f_{b_{i}}^{d_{i}}\right)$, because we do not need to use the Reducing Lemma at $b_{i}$.

Proof. By homotopy invariance we have $E O^{(n)}(f)=E O^{(n)}(g)$. So without loss of generality (by taking $g$ for $f$ ) we may assume that $b_{i} \in \operatorname{Fix}\left(\bar{f}^{d_{i}}\right)$ and $g$ is the same as $f$.

For each $b_{i} \in \xi$, let $\boldsymbol{F}_{\bar{f}, i}^{(n)}$ be the essential $n$-orbit class containing it. Clearly $\mathcal{E O}^{(n)}(f)=\bigcup_{i} p_{\mathcal{E}}^{-1}\left(\boldsymbol{F}_{\bar{f}, i}^{(n)}\right)$. So we only need to show

$$
\left|p_{\mathcal{E}}^{-1}\left(\boldsymbol{F}_{\bar{f}, i}^{(n)}\right)\right|=E O_{K}^{\left(m_{i}\right)}\left(f_{b_{i}}^{d_{i}}\right) \quad \text { when } \quad \operatorname{Fix}\left(\left(\bar{f}^{n}\right)_{*}^{b_{i}}\right)=\{1\} .
$$

In the following proof we shall drop the subscript $i$ from our notation. 
Suppose $b \in \xi$ is in the essential $n$-periodic point class $\boldsymbol{F}_{\bar{f}^{n}}$ which in turn is in the essential $n$-orbit class $\boldsymbol{F}_{\bar{f}}^{(n)}$. Since $d$ is the depth of $\boldsymbol{F}_{\bar{f}}^{(n)}, \boldsymbol{F}_{\bar{f} n}$ alone constitutes an essential $m$-orbit class $\boldsymbol{F}_{\bar{f}^{d}}^{(m)} \subset \boldsymbol{F}_{\bar{f}}^{(n)}$. Suppose $p_{\mathcal{E}}^{-1}\left(\boldsymbol{F}_{\bar{f}^{d}}^{(m)}\right) \neq \emptyset$.

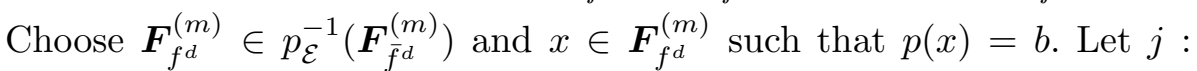
$F_{b} \hookrightarrow E$ be the inclusion of the fibre. We have the exact sequence

$$
1 \rightarrow \pi_{1}\left(F_{b}, x\right) / K \stackrel{j_{*}}{\rightarrow} \pi_{1}(E, x) \stackrel{p_{*}}{\rightarrow} \pi_{1}(B, b) \rightarrow 1 .
$$

Let ${ }_{K} \boldsymbol{F}_{f_{b}^{d}}^{(m)} \in \mathcal{E O}_{K}^{(m)}\left(f_{b}^{d}\right)$ be the mod $K$ essential orbit class containing $x$. Then by Proposition 2.3 we have a commutative diagram of exact sequences in the category of pointed sets:

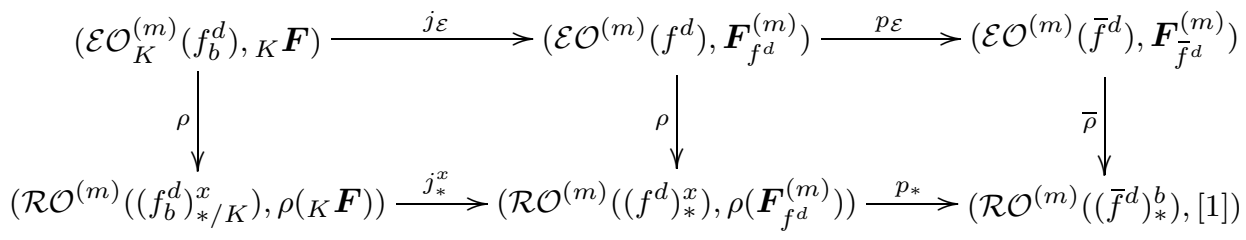

The notation ${ }_{K} \boldsymbol{F}$ stands for ${ }_{K} \boldsymbol{F}_{f_{b}^{d}}^{(m)}$ and [1] stands for $[1]_{\left(\bar{f}^{d}\right)_{*}^{b}}^{(m)}$. Note that here we regard $f^{d}$ as a self-map of the pair $\left(E, F_{b}\right)$. The base path is taken to be a path in $F_{b}$ from $x$ to $f^{d}(x)$, whose image in $B$ is the constant path at $b$. Hence the coordinate of $\boldsymbol{F}_{\bar{f}^{d}}^{(m)}$ is the $[1]_{\left(\bar{f}^{d}\right)_{*}^{b}}^{(m)}$ in the lower left corner.

When $\operatorname{Fix}\left(\left(\bar{f}^{d}\right)_{*}^{b m}\right)=\operatorname{Fix}\left(\left(\bar{f}^{n}\right)_{*}^{b}\right)=\{1\}$, Corollary 1.6 tells us $j_{*}^{x}$ is injective, and so $j_{\mathcal{E}}$ is injective. Since $p_{*}$ and $\sigma$ preserve essentiality, we have a commutative diagram of exact sequences in the category of pointed sets:

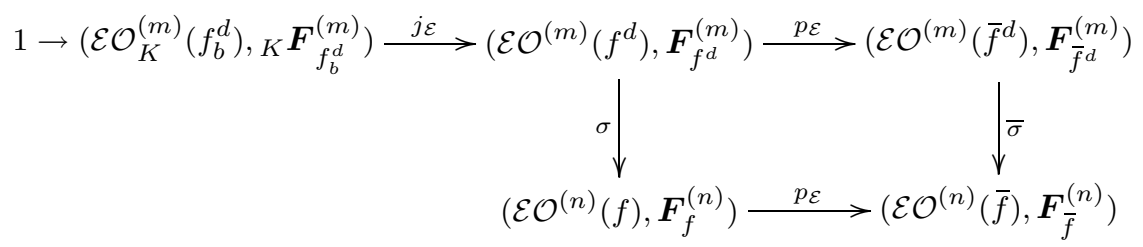

By Lemmas 1.12 and 1.7, $\sigma$ restricts to a bijection from $p_{\mathcal{E}}^{-1}\left(\boldsymbol{F}_{\bar{f}^{d}}^{(m)}\right)$ to $p_{\mathcal{E}}^{-1}\left(\boldsymbol{F}_{\bar{f}}^{(n)}\right)$. We thus get the desired equality $\left|p_{\mathcal{E}}^{-1}\left(\boldsymbol{F}_{\bar{f}}^{(n)}\right)\right|=E O_{K}^{(m)}\left(f_{b}^{d}\right)$.

Let $I E O^{(n)}(f)$ denote the number of irreducible essential $f$-orbit classes of $f$. Note that $I E O^{(1)}(f)=N(f)$, and $I E O^{(n)}(f)=(1 / n) N P_{n}(f)$ (as defined in [6, p. 69]). The proof of Theorem 2.4 also leads to the following consequence, which is comparable to [3, Theorem 3.4]. 
COROLLARY 2.5. Under the conditions of Theorem 2.4, we also have

$$
I E O^{(n)}(f)=\sum_{b_{i} \in \xi} I E O_{K}^{\left(m_{i}\right)}\left(g_{b_{i}}^{d_{i}}\right)
$$

The principal application is to fibrations over tori. It should be useful in calculations on nil and solvmanifolds.

Corollary 2.6. Suppose $p: E \rightarrow B$ is a fibration over a torus (of any dimension). Then for any fibre preserving map $f: E \rightarrow E$, the summation formulae of Theorem 2.4 and Corollary 2.5 hold true:

$$
E O^{(n)}(f)=\sum_{b_{i} \in \xi} E O_{K}^{\left(m_{i}\right)}\left(f_{b_{i}}^{d_{i}}\right), \quad I E O^{(n)}(f)=\sum_{b_{i} \in \xi} I E O_{K}^{\left(m_{i}\right)}\left(f_{b_{i}}^{d_{i}}\right) .
$$

Proof. For the torus map $\bar{f}$, the conditions of Theorem 2.4 are always satisfied, because torus maps are $n$-toral in the sense of [5, Definition 3.4 and Remark 3.6]. Moreover, if an essential orbit class of a torus map is reducible, it always reduces to an essential orbit class of the depth level, so we can assume $b_{i} \in \operatorname{Fix}\left(\bar{f}^{d_{i}}\right)$.

On the Klein bottle, Corollary 2.5 can be applied to establish the following interesting example (cf. [3, Example 4.1]). We omit the details.

ExAmple 2.7 (The Klein bottle). Represent the Klein bottle $K^{2}$ as the quotient $\mathbb{R}^{2} / G$, where $G$ is the group of automorphisms on $\mathbb{R}^{2}$ generated by $\alpha:(x, y) \mapsto(x, y+1)$ and $\beta:(x, y) \mapsto(x+1,-y)$.

Given any odd integer $r$, the map $(x, y) \mapsto(r x,-y)$ on $\mathbb{R}^{2}$ induces a well defined self-map $f$ of $K^{2}$. This $f$ is fibre preserving with respect to the fibration $S^{1} \hookrightarrow K^{2} \stackrel{p}{\rightarrow} S^{1}$, where $p$ is induced by the projection on the first factor. (It corresponds to the case $w=r$ and $u=-1$ in Example 1.16.) Note that $f$ induces a standard map $\bar{f}$ of degree $r$ on the base, and so $\bar{f}^{n}$ has exactly $N\left(\bar{f}^{n}\right)=\left|r^{n}-1\right|$ fixed points.

Then, for $n=2^{k}, k \geq 1$, we have

$$
E O^{(n)}(f)=I E O^{(n)}(f)=\frac{1}{n} N\left(f^{n}\right)=\frac{\left|r^{n}-1\right|}{n} .
$$

Acknowledgements. The authors are grateful to the referee for comments that helped improve the exposition.

\section{References}

[1] R. F. Brown, The Lefschetz Fixed Point Theorem, Scott, Foresman and Co., Glenview, IL, 1971.

[2] D. Ferrario, Computing Reidemeister classes, Fund. Math. 158 (1998), 1-18.

[3] P. R. Heath and E. C. Keppelmann, Fibre techniques in Nielsen periodic point theory on nil and solvmanifolds II, Topology Appl. 106 (2000), 149-167. 
[4] P. R. Heath, E. C. Keppelmann and P. N.-S. Wong, Addition formulae for Nielsen numbers and for Nielsen type numbers of fibre preserving maps, ibid. 67 (1995), $133-157$.

[5] P. R. Heath, R. Piccinini and C. You, Nielsen-type numbers for periodic points I, in: Topological Fixed Point Theory and Applications (Tianjin, 1988), B. Jiang (ed.), Lecture Notes in Math. 1411, Springer, Berlin 1989, 88-106.

[6] B. Jiang, Lectures on Nielsen Fixed Point Theory, Contemp. Math. 14, Amer. Math. Soc., Providence, RI, 1983.

[7] E. Spanier, Algebraic Topology, McGraw-Hill, New York, 1966.

Peking University

Beijing 100871, China

E-mail: jiangbj@math.pku.edu.cn

Korea University

Seoul 136-701, Korea

E-mail: woomh@korea.ac.kr
Mokwon University Daejeon 302-729, Korea E-mail: seoungho@mokwon.ac.kr

Received 17 February 2004;

in revised form 15 October 2004 